\title{
Incidence of impacted and supernumerary teeth-a radiographic study in a North Greek population
}

\author{
Anastasia Fardi ${ }^{1}$, Athena Kondylidou-Sidira ${ }^{2}$, Zaki Bachour ${ }^{3}$, Nikolaos Parisis ${ }^{4}$, Anastasios Tsirlis ${ }^{4}$
}

\begin{abstract}
${ }^{1}$ DDS, MSc. Department of Dentoalveolar Surgery, Implantology and Radiology, School of Dentistry, Aristotle University of Thessaloniki, Thessaloniki, Greece

${ }^{2}$ PHD, DDS. Lecturer, Department of Dentoalveolar Surgery, Implantology and Radiology, School of Dentistry, Aristotle University of Thessaloniki, Thessaloniki, Greece

${ }^{3}$ PHD, DDS. Research fellow, Department of Dentoalveolar Surgery, Implantology and Radiology, School of Dentistry, Aristotle University of Thessaloniki, Thessaloniki, Greece

${ }^{4}$ PHD, DDS. Associate Professor, Department of Dentoalveolar Surgery, Implantology and Radiology, School of Dentistry, Aristotle University of Thessaloniki, Thessaloniki, Greece
\end{abstract}

Correspondence:

Department of Dentoalveolar Surgery,

Implantology and Radiology, School of Dentistry, Aristotle University of Thessaloniki,

Thessaloniki 54124, Greece

nfardi@dent.auth.gr

Received:06/02/2010

Accepted: 04/06/2010

Fardi A, Kondylidou-Sidira A, Bachour Z, Parisis N, Tsirlis A. Incidence of impacted and supernumerary teeth-a radiographic study in a North Greek population. Med Oral Patol Oral Cir Bucal. 2011 Jan 1;16 (1):e5661.

http://www.medicinaoral.com/medoralfree01/v16i1/medoralv16ilp56.pdf

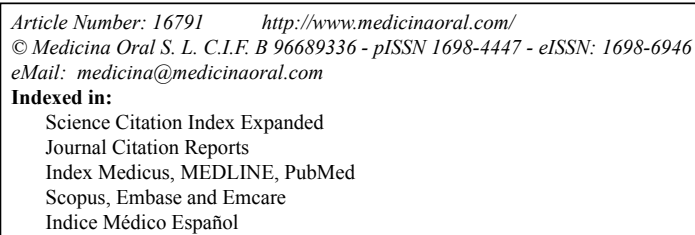

\begin{abstract}
Objectives: The aim of the present study was to investigate the prevalence of impacted and supernumerary teeth, apart from third molars.

Study design: This was a retrospective study of 1.239 panoramic radiographs taken of patients who presented to the Department of Dentoalveolar Surgery, Implantology and Radiology at the School of Dentistry of the Aristotle University of Thessaloniki, Greece between 1991 and 1999. The panoramic radiographs and dental records were reviewed in order to determine whether there were impacted or supernumerary teeth. Observations were also made on the space in dentition, corresponding to the position of each impacted tooth, the lack of space for tooth eruption, transmigration, retained primary teeth or prosthetic restoration.

Results: A total of 170 (13.7\%) patients presented with at least one impacted tooth. None of them had an impacted incisor. Impacted canines were the most prevalent dental anomaly $(8.8 \%)$, followed by impacted premolars (2.2\%). Supernumerary teeth $(1.8 \%)$ and impacted molars $(1 \%)$ were the least common anomalies. Among the $225 \mathrm{im}$ pacted teeth, the most frequently affected teeth were the canines $(59.6 \%)$, followed by premolars $(19.1 \%)$, and supernumerary teeth (15.1\%), while the incidence of impacted molars was substantially lower (6.2\%).

Conclusions: The most frequently impacted teeth were the maxillary canine, the second mandibular premolar and the second mandibular molar. The majority of the supernumerary teeth consisted of mesiodens. There was space in the dentition of each impacted tooth in $29.3 \%$ of the cases examined; there was a retained primary tooth in $25.1 \%$, and a prosthetic restoration had been constructed in $24 \%$. Insufficient space for the eruption of the impacted tooth and transmigration was observed in $17.3 \%$ and $4.2 \%$ of the cases, respectively.
\end{abstract}

Key words: Incidence, tooth unerupted, tooth supernumerary. 


\section{Introduction}

The eruption of permanent teeth represents a complex series of events, mostly genetically based, whereby eruptive movements of the tooth germ taking place at a predetermined time and route enable the tooth to find its antagonist at a predetermined occlusal plane. Apart from the eruption process, the successful development of permanent dentition involves the synchronized forward and lateral growth of both the maxilla and mandible, which compensates for the difference in size of the dentition in both bones. As the eruption process is so complex, it is not surprising that problems may arise, which lead to complications including tooth retardation or failure of eruption.

Failure of the eruption of permanent teeth is a common dental anomaly. When there is a clinical absence of one or several teeth, and the history indicates that they have not been extracted, then partial anodontia or tooth impaction should be considered. Radiographic examination may reveal impacted or supernumerary teeth. Developmental disturbances can be due to genetic or environmental factors, including physical and biological factors.

The prevalence of impacted and supernumerary teeth in different populations and ethnic groups has been the subject of several studies. The incidence of impacted teeth, excluding third molars, has been reported to vary between 5.6 to $18.8 \%(1-5)$. The present study was performed to determine the prevalence of impacted teeth in North Greek population.

\section{Materials and Methods}

This study comprises data from 1.239 patients who attended the Department of Dentoalveolar Surgery, Implantology and Radiology at the School of Dentistry of the Aristotle University of Thessaloniki in Greece between 1991 and 1999. Patients' dental records and radiographs were examined retrospectively in order to detect the following dental abnormalities: impacted canines, impacted premolars, impacted molars (except third molars), and supernumerary impacted teeth.

All radiographs were examined carefully by a single skilled dentist on a transparency projector under constant lighting conditions. A tooth that was prevented from erupting by a physical barrier, usually by other teeth, was defined as an impacted tooth. Impaction may also have resulted from the orientation of the tooth in any other position than vertical within the periodontal structures. Taking into account the mean eruption time, teeth were defined as impacted when they remained in the jaw two years after the respective mean age of tooth eruption (6). A supernumerary tooth is an extra tooth, which may be erupted or unerupted, and either resembles or is unlike the other teeth in the group to which it belongs. Its presence may cause malposition of the adjacent teeth or prevent their eruption. In addition to the presence of the impacted teeth, a detailed description was given of the presence of any primary tooth that was present in place of the impacted permanent tooth, the lack of space for the impacted tooth, the presence of prosthetic rehabilitation, and the possible transmigration of the impacted tooth. After the examination of the patient records, patients who exhibited one or more of the following pathological situations were excluded from the study:

- Any disease, trauma or fracture of the jaw that might have affected the normal growth of permanent dentition.

- Any hereditary diseases or syndromes such as Down's syndrome or cleidocranial dysostosis.

Data were gathered and analyzed using the SPSS statistical package (version 12 software, Chicago, Illinois). The differences between the groups were tested using the Chi-square test, Mann-Whitney test and a one-way analysis of variance (ANOVA). The level of significance was set at $5 \%(\mathrm{p}<0.05)$.

\section{Results}

Of 1.239 patients, 593 were male (47.9\%) and 646 were female $(52.1 \%)$; the sex ratio was therefore $1: 1.1$. The mean age was $33 \pm 15.71$ years, ranging from 7 to 92 years. At least one impacted tooth was detected in 170 of the patients $(13.7 \%)$. The total number of impacted teeth among the affected 170 patients was 225 , with an average number of impacted teeth per person of 1.3. A total of 106 impacted teeth were found in 78 males $(45.9 \%)$ and 119 were in 92 females (54.1\%). The incidence of impacted teeth did not differ significantly between the sexes $(p>0.05)$.

Table 1. Prevalence of patients with an impacted tooth according to gender and the number of impacted teeth.

\begin{tabular}{|l|l|l|c|l|}
\hline \multicolumn{5}{|c|}{ Number (\%) of patients with impacted tooth } \\
\hline & One & Two & $\geq$ Three & Total \\
\hline Male & $59(34.7)$ & $14(8.2)$ & $5(2.9)$ & $\mathbf{7 8 ( 4 5 . 9 )}$ \\
\hline Female & $70(41.2)$ & $17(10.0)$ & $5(2.9)$ & $\mathbf{9 2}(\mathbf{5 4 . 1 )}$ \\
\hline Total & $\mathbf{1 2 9}(\mathbf{7 5 . 9})$ & $\mathbf{3 1 ( 1 8 . 2 )}$ & $\mathbf{1 0}(\mathbf{5 . 3})$ & $\mathbf{1 7 0}(\mathbf{1 0 0 )}$ \\
\hline
\end{tabular}




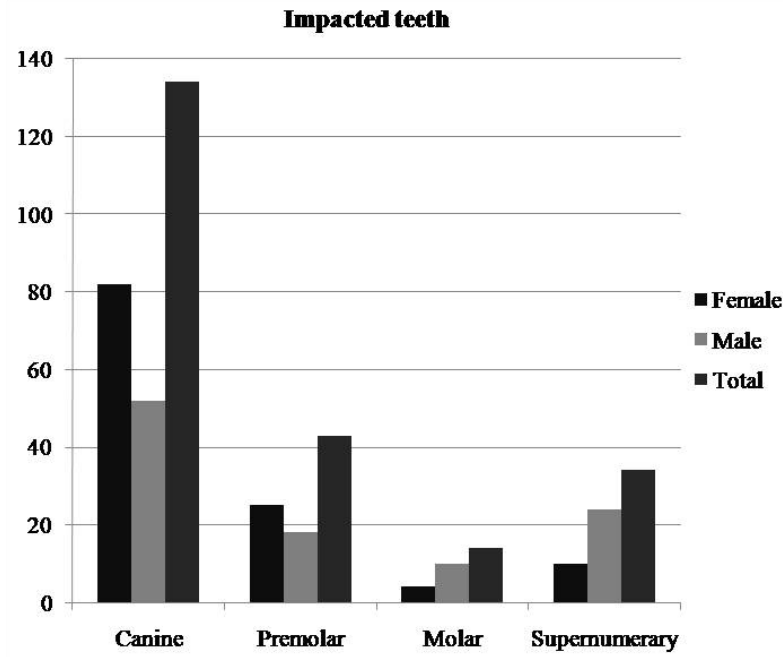

Fig. 1. Distribution of patients with impacted teeth according to gender and tooth type.

A total of 129 patients had one impacted tooth, 31 patients had two impacted teeth, and the remaining ten patients had at least three or more impacted teeth (Table 1). The number of impacted teeth was not associated with gender $(p>0.05)$. Impacted canines were the most prevalent dental anomaly $(8.8 \%)$, followed by impacted premolars $(2.2 \%)$. Supernumerary teeth $(1.8 \%)$ and impacted molars $(1 \%)$ were the least common anomalies. None of the patients presented with an impacted incisor. A gender-specific increased incidence of impacted teeth was not detected $(\mathrm{p}>0.05)$.

The sample of 170 patients was further divided into four groups according to the type of impacted tooth. Regarding the location of the impacted teeth, they were classified according to which jaw they were in. Since no statistical differences were observed relating to the jaw side and tooth impaction $(\mathrm{p}>0.05)$, the localiza- tion of the right/left quadrant was not taken into consideration. The distribution of impacted teeth according to gender and type is shown in fig. 1. Among the 225 impacted teeth, the most frequently affected teeth were the canines (59.6\%), followed by premolars (19.1\%), and supernumerary teeth $(15.1 \%)$, while the incidence of impacted molars was substantially lower (6.2\%). There was not a significant relationship between the type of impacted tooth and gender $(p>0.05)$.

Impacted canines were diagnosed in 109 cases (8.8\%). In 104 cases (8.4\%), the impacted canine was located in the maxilla, while seven patients displayed at least one canine in the mandible (Fig. 2). Bilateral canine impaction was recorded in 19 subjects. Among them, only one subject had both mandibular canines impacted. Full impaction of all four canines was found in one patient. With regards to the patients with impacted premolars, 27 patients were found to have at least one affected premolar $(2.2 \%)$. The most commonly affected tooth was the second mandibular premolar, followed by the second maxillary premolar. Nine cases were diagnosed to have more than two impacted premolars (Fig. 2).

Of the 170 patients with impacted teeth, thirteen displayed impacted molars (1\%). The mandibular second molar was the most frequently observed affected tooth. The remaining molars were equally distributed with equal percentages (Fig. 3). One case had two impacted molars.

Fig. 3 summarizes the distribution of the 23 patients with impacted supernumerary teeth. Their mean age is significantly younger than patients of the other categories, being $17.2 \pm 10.8$ years, and the median age was 13 years. A total of 15 patients had supernumerary teeth located in the premaxilla, of which 13 displayed mesiodens and only two had supernumerary teeth located in the lateral incisor region. Supernumerary teeth in the premolar region and fourth molars were equally distributed in eight patients, respectively.
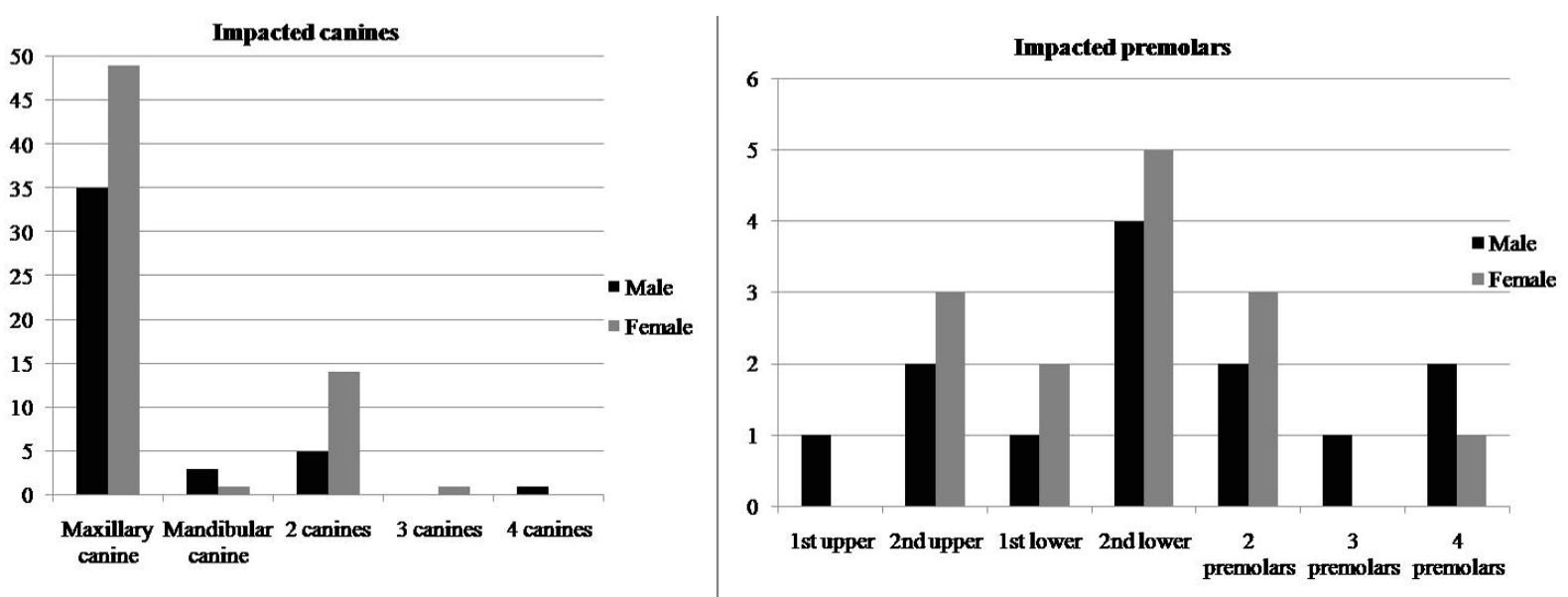

Fig. 2. Distribution of patients with impacted canines and impacted premolars according to gender. 

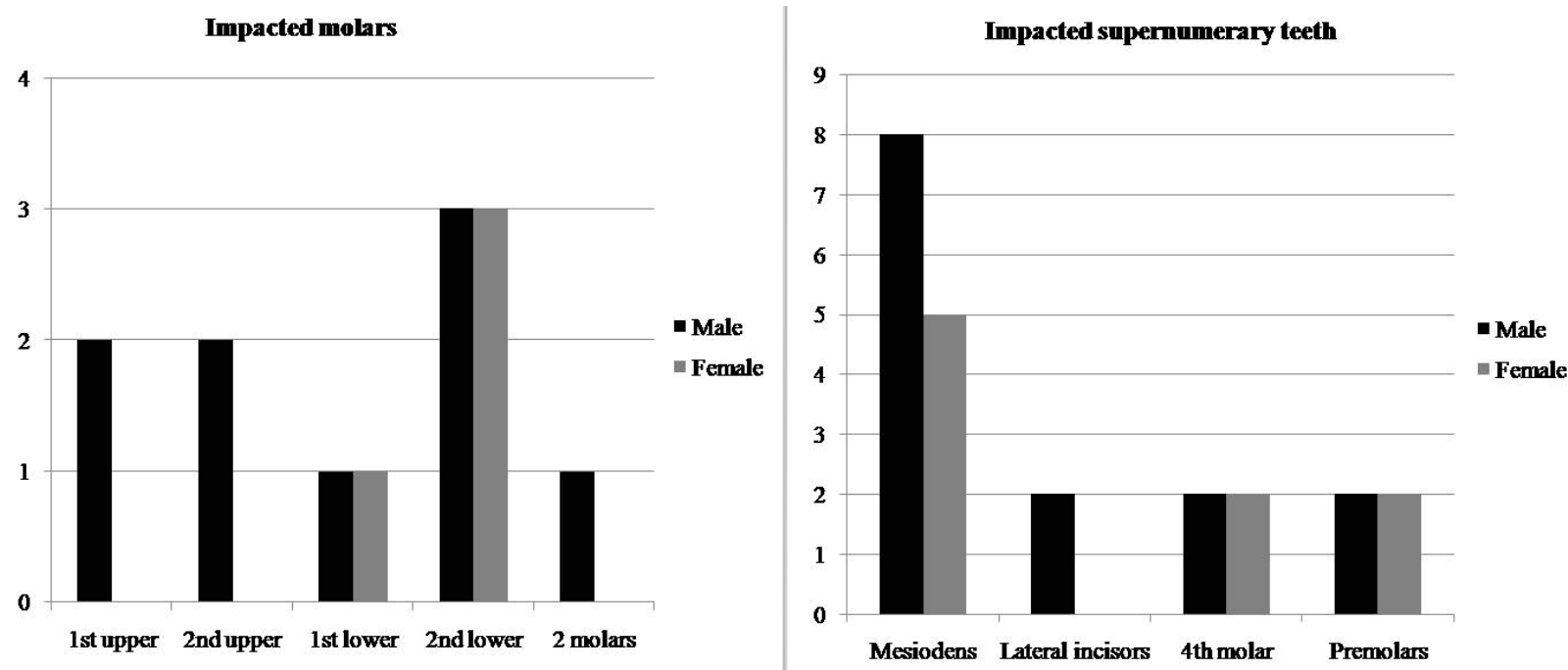

Fig. 3. Distribution of patients with impacted molars according to gender and distribution of impacted supernumerary teeth according to their location and the patients' gender.

Regarding the space in dentition corresponding to the position of each impacted tooth, it was observed that there was space in the dentition in $29.3 \%$ of the cases examined, the primary tooth was present in $25.1 \%$ of cases, and prosthetic restoration had been constructed in $24 \%$ of cases. Insufficient space for the eruption of the impacted tooth and transmigration was observed in $17.3 \%$ and $4.2 \%$ of the cases, respectively (Table 2 ).

\section{Discussion}

The present data indicate that the prevalence of tooth impaction in North Greek population is similar to those reported in other studies while other studies report different rates. Our data shows the incidence of tooth impaction to be $13.7 \%$, and this falls within the range of 5.6-18.8\% reported in other studies. To compare the prevalence found in this study with the different frequencies reported in the dental literature from other studied populations, one should consider the methodology used for detection of these anomalies as well as the clinical differences of the epidemiological studies, including sample selection, definition of impacted tooth and the age range of subjects.
It is not easy to choose an appropriate sample to examine the frequency of impacted teeth. To determine the actual prevalence of tooth impaction, a representative and randomized sample of the general population is required. Undoubtedly, it is not straightforward to collect such information, as exposing patients to radiation for research purposes conflicts with medical ethics. The most common practical approach is to examine radiographs from specific populations, which will inevitably involve the risk of bias in the data analysis.

Taking into account the source of the analyzed data, which were derived from our Department, the large age range of the examined sample and the limited exclusion criteria, one might consider that the results of this study are not representative of the general population. However, the primary aim of this study was to investigate the frequency of impacted teeth in patients who attend our Department. The low age limit of our sample is due to the inclusion of patients having supernumerary teeth, which are typically diagnosed during childhood.

We found that canines were the most commonly impacted teeth, which is in agreement with other studies. In our study, the presence of an impacted canine was

Table 2. Distribution of impacted teeth according to the space in dentition corresponding to the position of each tooth.

\begin{tabular}{|l|c|c|c|c|c|}
\hline & $\begin{array}{c}\text { Primary } \\
\text { tooth }\end{array}$ & Space & $\begin{array}{c}\text { Prosthetic } \\
\text { rehabilitation }\end{array}$ & $\begin{array}{c}\text { Lack of } \\
\text { space }\end{array}$ & Transmigration \\
\hline Impacted tooth & & & & & \\
\hline Canines & $36(18.8)$ & $33(17.2)$ & $45(23.5)$ & $20(10.5)$ & $0(0)$ \\
\hline Premolars & $12(6.3)$ & $15(7.8)$ & $1(0.5)$ & $11(5.8)$ & $4(2.1)$ \\
\hline Molars & $0(0)$ & $8(4.2)$ & $0(0)$ & $2(1)$ & $4(2.1)$ \\
\hline Total (\%) & $\mathbf{4 8 ( 2 5 . 1 )}$ & $\mathbf{5 6 ( 2 9 . 3 )}$ & $\mathbf{4 6 ( 2 4 )}$ & $\mathbf{3 3 ( 1 7 . 3 )}$ & $\mathbf{8 ( 4 . 2 )}$ \\
\hline
\end{tabular}


found in $8.8 \%$ of cases, which is significantly higher than the $3.58 \%$ incidence reported by Aydin et al. (7). In one study, 4.898 Saudi patients aged 13 years or older were examined, and the results indicated that $3.6 \%$ had at least one impacted cuspid (8). Another study analysed 1.858 children who presented for orthodontic treatment, and the results revealed 101 cases of impacted cuspids $(5.43 \%)(9)$. The authors of the same study stated that the frequency of impacted canines was dependant on the population studied (9). The results of our study showed that $8.4 \%$ maxillary canines were impacted, a result that is much higher than the $1-3 \%$ incidence reported for other various populations $(1-4,10,11)$. The different results from these studies may arise from racial differences and differences in the methodology of the study. As reported previously, the rarity of impacted canines in the mandible was confirmed in this study $(1,2)$. Indeed, most of the impacted canines were located in the maxilla, which had also been established as the predominant location by others (1). Impacted canines of the mandible, of which there were only six cases in our study, are very rare in the general population. Grover and Lorton examined 5,000 radiographs and found 142 examples of impacted canines in the maxilla $(2.84 \%)$ and only 11 in the mandible ( $0.22 \%)$ (2). In one study that included 1.000 Turkish patients, the incidence of maxillary canine impaction was $2.9 \%$, while the incidence of impacted mandibular canines was $0.3 \%$ (12). In another study, Shah et al. detected only eight impacted canines in the mandible among 7.886 patients $(0.10 \%)$ (5). Although they are not a common occurrence, bilaterally impacted canines were detected in $19.2 \%$ of patients with impacted canines, a value that falls within the range of $17-45 \%$ reported by Jacobs (13).

Although there was no difference in the sex distribution for impacted canines in our study, the male to female prevalence rate ratios was 1:2.4. This result is within the range from 1:1.3 to 1:3.2 reported in the literature, indicating a higher prevalence of impacted canines among females (13). Additionally, it has been observed that the incidence of impacted canines depends on the population studied, and the ratio of impacted canines between European to Asian patients is 2:1 (10). It should be noted here that none of the impacted canines were transmigrated, which is in agreement with other authors' opinions, who state that transmigration is an extremely rare anomaly (14).

Very limited information is available regarding impacted premolars. From the few studies available, which have examined thousands of medical records, it seems that premolar impaction is rare, with the corresponding frequency ranging from $2.1-2.7 \%(1,3,4)$. These results are comparable to the incidence of $2.2 \%$ reported in the present study. Although one study showed that first maxillary premolars remained impacted more frequent- ly (11), our study indicated that the second premolar of the lower jaw was the most frequently impacted tooth, followed by the second premolar of the maxilla.

The literature review of impacted molars demonstrates that this is a very rare dental abnormality, which is consistent with the low incidence found in the present study (15). As was the case in other studies, we observed increased percentages in male patients, which suggests a genetic component $(15,16)$.

Supernumerary teeth are not an uncommon finding in dental practice, occurring in $0.1-3.8 \%$ of the population (17-19). The reported prevalence of supernumerary impacted teeth $(1.8 \%)$ in the present study falls within this range. The incidence of the supernumerary teeth in different populations was the subject of several studies. Bäckman and Wahlin clinically examined 739 Caucasian children and found 14 cases of at least one supernumerary tooth. They also noted that the majority of the supernumerary teeth were mesiodens (17). Salem conducted a survey of 2,393 Saudi Arabian children and found that only $0.5 \%$ had supernumerary teeth (18).

Many studies indicate a higher prevalence of supernumerary teeth amongst males. Contrary to the 2:1 ratio between males and females reported in Caucasians (19), in the present study the sex ratio was much lower, being 1.4:1 in favor of males. However, this is in accordance with the 1.3:1 ratio reported by Luten (19), 1.82:1 reported by Ferrés-Padró et al. (20) and 1.2:1 reported by Salcido-García et al. (21).

The majority of supernumerary teeth found in the present study were located in the premaxilla. The remaining teeth were located in the mandibular premolar region, the maxillary molar region as fourth molars, and the maxillary lateral incisor region. Luten's study suggested the following order of decreasing frequency: upper lateral incisors, mesiodens, upper central incisors followed by bicuspids (19). Results of a study investigating the frequency of supernumerary teeth in a Mexican population suggested that mesiodens was the most frequent, followed by premolars, lateral incisors and 4th molars (21). However, multiple supernumerary teeth may appear either in the premolar (20) or in the premaxillary zone (22).

Our data show that the prevalence of tooth impaction is a common dental anomaly. The early recognition of tooth impaction is very important from a therapeutic point of view. Impacted teeth result in many complications and their early detection is imperative. The results of this study were similar to the data reported in other studies, while the dissimilarities may be attributed to the sample selection, method of the study and area of patient selection, which suggest racial and genetic differences. 


\section{References}

1. Kramer RM, Williams AC. The incidence of impacted teeth. A survey at Harlem hospital. Oral Surg Oral Med Oral Pathol. 1970;29:237-41.

2. Grover PS, Lorton L. The incidence of unerupted permanent teeth and related clinical cases. Oral Surg Oral Med Oral Pathol. 1985;59:420-5.

3. Thilander B, Myrberg N. The prevalence of malocclusion in Swedish schoolchildren. Scand J Dent Res. 1973;81:12-21.

4. Dachi SF, Howell FV. A survey of 3,874 routine full-month radiographs. II. A study of impacted teeth. Oral Surg Oral Med Oral Pathol. 1961;14:1165-9.

5. Shah RM, Boyd MA, Vakil TF. Studies of permanent tooth anomalies in 7,886 Canadian individuals. I: impacted teeth. Dent J. 1978;44:262-4.

6. Wedl JS, Danias S, Schmelzle R, Friedrich RE. Eruption times of permanent teeth in children and young adolescents in Athens (Greece). Clin Oral Investig. 2005;9:131-4.

7. Aydin U, Yilmaz HH, Yildirim D. Incidence of canine impaction and transmigration in a patient population. Dentomaxillofac Radiol. 2004;33:164-9.

8. Zahrani AA. Impacted cuspids in a Saudi population: prevalence, etiology and complications. Egypt Dent J. 1993;39:367-74.

9. Rózsa N, Fábián G, Szádeczky B, Kaán M, Gábris K, Tarján I. [Prevalence of impacted permanent upper canine and its treatment in 11-18-year-old orthodontic patients]. Fogorv Sz. 2003;96:65-9.

10. Peck S, Peck L, Kataja M. The palatally displaced canine as a dental anomaly of genetic origin. Angle Orthod. 1994;64:249-56.

11. Roberts-Harry D, Sandy J. Orthodontics. Part 10: Impacted teeth. Br Dent J. 2004;196:319-27.

12. Sağlam AA, Tüzüm MS. Clinical and radiologic investigation of the incidence, complications, and suitable removal times for fully impacted teeth in the Turkish population. Quintessence Int. 2003;34:53-9.

13. Jacobs SG. The impacted maxillary canine. Further observations on aetiology, radiographic localization, prevention/interception of impaction, and when to suspect impaction. Aust Dent J. 1996;41:310-6.

14. Torres-Lagares D, Flores-Ruiz R, Infante-Cossío P, GarcíaCalderón M, Gutiérrez-Pérez JL. Transmigration of impacted lower canine. Case report and review of literature. Med Oral Patol Oral Cir Bucal. 2006;11:E171-4.

15. Baccetti T. Tooth anomalies associated with failure of eruption of first and second permanent molars. Am J Orthod Dentofacial Orthop. 2000;118:608-10.

16. Valmaseda-Castellón E, De-la-Rosa-Gay C, Gay-Escoda C. Eruption disturbances of the first and second permanent molars: results of treatment in 43 cases. Am J Orthod Dentofacial Orthop. 1999;116:651-8.

17. Bäckman B, Wahlin YB. Variations in number and morphology of permanent teeth in 7-year-old Swedish children. Int J Paediatr Dent. 2001;11:11-7.

18. Salem G. Prevalence of selected dental anomalies in Saudi children from Gizan region. Community Dent Oral Epidemiol. 1989;17:162-3.

19. Luten JR Jr. The prevalence of supernumerary teeth in primary and mixed dentitions. J Dent Child. 1967;34:346-53.

20. Ferrés-Padró E, Prats-Armengol J, Ferrés-Amat E. A descriptive study of 113 unerupted supernumerary teeth in 79 pediatric patients in Barcelona. Med Oral Patol Oral Cir Bucal. 2009;14:E146-52.

21. Salcido-García JF, Ledesma-Montes C, Hernández-Flores F, Pérez D, Garcés-Ortíz M. Frequency of supernumerary teeth in Mexican population. Med Oral Patol Oral Cir Bucal. 2004;9:407-9; 403-6.

22. Yagüe-García J, Berini-Aytés L, Gay-Escoda C. Multiple supernumerary teeth not associated with complex syndromes: a retrospective study. Med Oral Patol Oral Cir Bucal. 2009;14:E331-6. 